\title{
Conditional independence in dynamic networks
}

\author{
Jürgen Lerner*, Natalie Indlekofer, Bobo Nick, Ulrik Brandes \\ Department of Computer \& Information Science, University of Konstanz, Germany
}

\begin{abstract}
A B S T R A C T
Given a longitudinal network observed at time points $t_{1}<\cdots<t_{T}$, tie changes that happen in the interval $\left(t_{h}, t_{h+1}\right)$ typically depend on the networks at $t_{1}, \ldots, t_{h}$. In this article we deal with the question

whether changes within one interval mutually depend on each other or whether they are conditionally independent, given the previously observed networks. Answering this question for given data is of high practical relevance since, if the conditional independence assumption is valid, network dynamics can be modeled with simple and computationally efficient statistical techniques for independent observations. Consequently, we propose a framework to systematically compare conditional independence models with more general models that are specifically designed for social network data. Our results suggest that conditional independence models are inappropriate as a general model for network evolution and can lead to distorted substantive findings on structural network effects, such as transitivity. On the other hand, the conditional independence assumption becomes less severe when inter-observation times are relatively short.
\end{abstract}

Keywords:

Network modeling

ERGM

SAOM

Conditional independence

\section{Introduction}

One of the most universal characteristics of network data is the property that dyadic observations are typically not independent. For instance, if two actors have a common friend, the probability of a friendship tie among them is often highly increased. Likewise, actors often show a tendency to connect with those that are already well connected in the network. Such non-independence among observations can make the design of valid network models a challenging task. In a quite general class of models (Frank \& Strauss, 1986), a dyadic observation might depend on the presence or absence of other incident ties. Direct computation of probabilities in such network models is computationally intractable for all but the smallest networks. Sophisticated stochastic approximation techniques exist but are still computationally expensive and might suffer from model degeneracy (Hunter \& Handcock, 2006; Snijders, 2002).

The situation becomes much easier if complete and finegrained information about the ordering and timing of tie changes is available, for instance, if dyadic observations do not encode relational states but continuously observed relational events between actors. Recently proposed models for event networks (Brandes, Lerner, \& Snijders, 2009; Butts, 2008) assume that events

\footnotetext{
This research has been supported in part by DFG under grant $\mathrm{Br}$ 2158/3-2 and the University of Konstanz under grant FP 665/10.

* Corresponding author.

E-mail addresses: lerner@inf.uni-konstanz.de (J. Lerner),

ulrik.brandes@uni-konstanz.de (U.Brandes).
}

are conditionally independent of each other and can be estimated by computationally more efficient algorithms.

Empirical longitudinal network data often fall between these two extremes (of tie changes being completely (un)ordered) in the sense that there is some, but not perfect, time information; examples include panel data about relational states and intervalcensored relational event data. The former arises from data collection strategies where a dynamically evolving network whose ties encode relational states (such as friendship) has been observed at two or more discrete points in time. The network at a particular observation point is assumed to result from the preceding one and a sequence of unobserved tie-change events. Similarly, intervalcensored relational event data (such as email correspondence with timestamps given by the day) does not encode the order of events that happen within the same time interval. In both variants it is still reasonable to assume that past events are not influenced by future events; however, since it is partially unknown which event precedes which, it is questionable whether mutual dependencies can be excluded from the models. The goal of this paper is to shed light on this question by systematically comparing simple models that assume conditional independence of tie-change events with more involved models that do not. Furthermore, we treat the question whether substantive findings about hypothetical network effects, such as transitivity, are affected by the (potentially invalid) assumption of conditional independence.

There are several sources of motivation for conducting this study. The first (rather general and intrinsic) argument is that we simply want to learn as much as possible about the dependence structure in empirical network data. More practically, we observe that conditional independence of tie-change events within observation periods is often (sometimes implicitly) assumed in published research (see, e.g., (Crescenzi, 2007; Hanneke, Fu, \& Xing, 
2010; Maoz, Terris, Kuperman, \& Talmud, 2007) for the case of panel data about relational states and (Brandes et al., 2009) for the case of interval-censored relational event data). It is thus relevant to test the validity of this assumption and to tackle the question whether it affects the reported findings. Steglich, Snijders, and Pearson (2010) argue against models that assume independence of tie-change events within observation intervals. It is not our goal to challenge this claim but rather to quantify the implications of assuming independence when analyzing given longitudinal network data. While it can be expected that conditional independence models are inappropriate as a general model for network evolution, it is possible that the independence assumption becomes less severe when the observation intervals are relatively short compared to the usual rate of change of the analyzed relation. Since conditional independence models are computationally much simpler and lead to more stable estimation algorithms, they are preferable for practical reasons if the danger of drawing invalid conclusions can be ruled out.

We formalize the conditional independence assumption in Section 2. In Section 3, we recall two established model frameworks that are used in the comparative study of this paper. Subsequently, Section 4 introduces two datasets, differing among others in the observation frequency, that we take in our analysis and presents the precise specification of the models to be compared. Findings are presented and discussed in Section 5 and we end with a summary of the main insights and indicate future work.

\section{Network dynamics}

For clarity of exposition, we restrict ourselves to panel data about networks of relational states but briefly note in the conclusion that interval-censored relational event data can be treated with very similar methods.

\subsection{Basic notation}

The input data (i. e., the observation) consists of a number $T \geq$ 2 of binary matrices $y^{(1)}, \ldots, y^{(T)}$ of order $n \times n$, where $n$, the number of actors, is kept constant over time. These matrices are assumed to be realizations of a continuous-time random process $Y^{(t)} \in\{0,1\}^{n \times n}, t \in \mathbb{R}$, at observation times $t_{1}<\cdots<t_{N}$. The observation that the $i, j$-th entry of $y$ equals one at time $t_{h}$ (which is denoted by $y_{i, j}^{(h)}=1$ ) encodes that there is a tie from actor $i$ to actor $j$ at that time; else $y_{i, j}^{(h)}=0$. We describe the following for networks that are directed (i.e., $y_{i, j}^{(h)}$ might be different from $y_{j, i}^{(h)}$ ) and have no loops (i.e., $y_{i, i}^{(h)}=0$ ). Adaptation to undirected networks or networks with loops is straightforward. The observation does additionally encode $q \geq 0$ actor-level covariates $v=\left(v_{1}, \ldots, v_{q}\right) \in$ $\mathbb{R}^{q \times n}$ and $q^{\prime} \geq 0$ dyad-level covariates $w=\left(w_{1}, \ldots, w_{q^{\prime}}\right) \in$ $\mathbb{R}^{q^{\prime} \times n \times n}$. These covariates might influence tie-change probabilities; on the other hand, we take the observed covariates as given and do not model their evolution. We assume that the covariates are observed at the observation times $t_{1}, \ldots, t_{T-1}$ and remain constant within the semi-open intervals $\left[t_{h-1}, t_{h}\right)$. For keeping notation concise, we often do not explicitly write dependence on covariates; for instance the conditionally probability $P\left(Y^{\left(t_{h}\right)}=\right.$ $\left.y^{(h)} \mid y^{(h-1)}\right)$ is short-hand for $P\left(Y^{\left(t_{h}\right)}=y^{(h)} \mid y^{(h-1)}, v^{(h-1)}, w^{(h-1)}\right)$.

Further, we denote for any network $y$ and pair of actors $(i, j)$ by $y^{-i j}$ the network resulting from $y$ by setting the $i, j$ 'th entry to zero and by $y^{+i j}$ the network resulting from $y$ by setting this entry to one. More generally, $y^{-i j}$ refers to flipping a tie $y_{i j}$ into its opposite $1-y_{i j}$.

\subsection{Conditional independence}

Assume that we are given a continuous-time random process $Y^{(t)} \in\{0,1\}^{n \times n}, t \in \mathbb{R}$, and a fixed sequence of observation times
$t_{1}<\cdots<t_{T}$, as described above. The conditional independence assumption states that, for all $h \geq 2$, the conditional distribution of the network $y^{(h)}$, given the networks $y^{(1)}, \ldots, y^{(h-1)}$, is the product of the conditional probabilities of the individual ties. Formally, for all $h \geq 2$ it is

$$
\begin{aligned}
& P\left(Y^{\left(t_{h}\right)}=y^{(h)} \mid y^{(1)}, \ldots, y^{(h-1)}\right) \\
& \quad=\prod_{i \neq j} P\left(Y_{i j}^{\left(t_{h}\right)}=y_{i j}^{(h)} \mid y^{(1)}, \ldots, y^{(h-1)}\right) .
\end{aligned}
$$

Thus, in a conditional independence model, the probability of a tie between $i$ and $j$ at time $t_{h}$ might depend on the presence or absence of another tie, say $(i, k)$, at time points $t_{h^{\prime}}, h^{\prime}<h$, but not on whether the tie on $(i, k)$ was created or dissolved in the interval $\left(t_{h-1}, t_{h}\right)$. As a basic example, models that include only covariates as explanatory variables satisfy the conditional independence assumption.

\subsection{Lagged network statistics}

To allow for models that include dependence among dyads but still satisfy the conditional independence assumption, we introduce another class of network statistics resembling dyadic covariates but having a fundamentally different interpretation.

A lagged (dyadic) network statistic is defined to be a family of $T-1$ real $n \times n$ matrices $x^{(1)}, \ldots, x^{(T-1)}$ that are deterministic functions of the observed networks $y^{(1)}, \ldots, y^{(T-1)}$, respectively. Similarly to covariates, the lagged network statistics are kept constant in the intervals between observations $\left[t_{h-1}, t_{h}\right.$ ) and are assumed to influence the evolution of ties within these intervals. For example, a lagged network statistic at time $t_{h-1}$ might encode for each pair of actors $(i, j)$ the number of common friends of $i$ and $j$ in the observed network $y^{(h-1)}$; a high value of this statistic might turn out to increase the probability that $i$ and $j$ create or sustain a friendship tie with each other in the time interval following $t_{h-1}$. Note that such lagged network statistics can imply statistical dependence among different dyads only in a restricted form: tie changes in $\left(t_{h-1}, t_{h}\right)$ depend via lagged network statistics on other change events (potentially on different dyads) only if these happened before or at $t_{h-1}$. Thus, a model that allows dyadic dependence only via lagged network statistics falls into the class of conditional independence models; it can express mutual dependence among dyads but excludes mutual dependence among individual change events.

\subsection{Illustrating example}

For an illustrating example, consider the two networkevolution scenarios shown in Fig. 1. The evolution in the upper row could be explained by a conditional independence model that includes a transitivity effect: the lagged network statistic that encodes for each pair of actors $(i, j)$ the number of directed twopaths from $i$ to $j$ assigns the value one to $(A, B)$ and zero to any other pair. Including this statistic in the specification of the tie probabilities can lead to an increased probability of the newly created tie $(A, B)$.

The situation is very different in the bottom row of Fig. 1 . There the same lagged network statistic that counts directed twopaths is zero for all pairs, implying that this statistic does not yield an increased probability of the tie $(A, B)$ - even though $(A, B)$ closes a transitive triangle in the network observed at $t_{h}$. In contrast, models that do not rely on conditional independence could interpret the network evolution in the bottom row as a support for transitive closure (assuming that this change pattern has been frequently observed in the data). Two such model classes are recalled in the next section. 


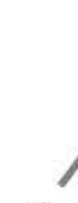

A
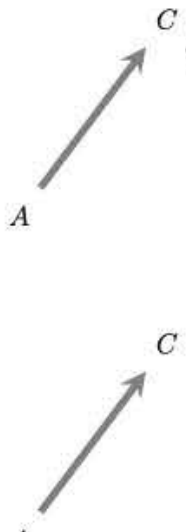

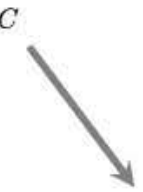

$B$
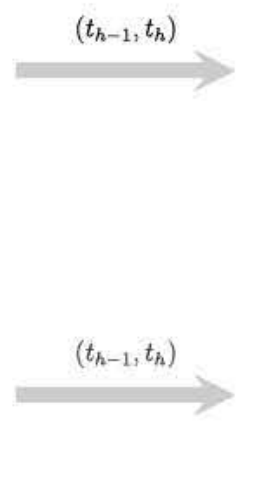
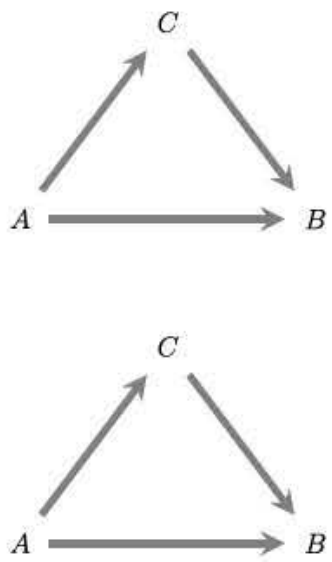

Fig. 1. Two different change scenarios in which the networks observed at $t_{h-1}$ (left) evolve into the networks observed at $t_{h}$ (right). Note that a conditional independence model could attribute the evolution in the top row to transitivity since the newly created tie $(A, B)$ closes a two-path that was present at the first time point.

\section{Models}

We compare conditional independence models with more general models in two established frameworks for network modeling. The first model framework is a temporal extension of exponential random graph models (Robins, Snijders, Wang, Handcock, \& Pattison, 2007) - like the one proposed by Hanneke et al. (2010) as well as Cranmer and Desmarais (2011) - the second comprises the stochastic actor-oriented models from Snijders (2001, 2005).

From now on we make the (Markovian) assumption that the network evolution in the interval $\left(t_{h-1}, t_{h}\right)$ is stochastically determined by the network $y^{(h-1)}$ but conditionally independent of the previous networks $y^{(1)}, \ldots, y^{(h-2)}$. Formally, for all $h \geq 2$ it is

$P\left(Y^{\left(t_{h}\right)}=y^{(h)} \mid y^{(h-1)}\right)=P\left(Y^{\left(t_{h}\right)}=y^{(h)} \mid y^{(1)}, \ldots, y^{(h-1)}\right)$.

This Markovian assumption has nothing to do with the conditional independence assumption that is the focus of this paper. While conditional independence could also be investigated without the Markov assumption, this would blow up the models and notation.

Further, the proposed models will allow parameters $\theta_{\ell}^{(h)}$ to depend on time step $h$ (i.e., the model is heterogeneous over time). Specifying a time-homogeneous model is straightforward by requiring that $\theta^{(h)}=\theta^{\left(h^{\prime}\right)}$ for all pairs of observation points $h, h^{\prime} \geq 2$.

\subsection{Exponential random graph models (ERGMs)}

A random graph model belongs to the class of exponential random graph models (Robins et al., 2007) if the probability of a network $y$ is specified by

$P(Y=y)=\frac{1}{z(\theta)} \cdot \exp \left(\sum_{\ell=1}^{k} \theta_{\ell} \cdot s_{\ell}(y)\right)$,

where the $s_{\ell}$ are statistics mapping a network $y$ to a real number, the $\theta_{\ell} \in \mathbb{R}$ are parameters modeling the influence of the statistics on the probability of the network, and $z(\theta)$ is a normalization constant ensuring that the probabilities of all possible networks sum up to one. Appropriate statistics introduce dependence among dyads. For instance, specifying an ERGM with statistics that count the number of edges, triangles, and $k$-stars $(k=2, \ldots, n-2)$ in the network yields an ERGM in which two dyads that share a common actor mutually depend on each other.

Bringing in a temporal component into the ERGM class is straightforward and is based on the concept of lagged network statistics introduced earlier. More specifically, given an observed sequence of networks $\left(y^{(1)}, \ldots, y^{(T)}\right)$, the conditional probability $P\left(Y^{\left(t_{h}\right)}=y^{(h)} \mid y^{(h-1)}\right)$ is modeled as in Eq. (3) where we allow the statistics to be functions of both networks $y^{(h)}$ and $y^{(h-1)}$. In formulas, for all $h \geq 2$

$$
\begin{aligned}
& P\left(Y^{\left(t_{h}\right)}=y^{(h)} \mid y^{(h-1)}\right) \\
& \quad=\frac{1}{z\left(\theta^{(h)}\right)} \cdot \exp \left(\sum_{\ell=1}^{k} \theta_{\ell}^{(h)} \cdot s_{\ell}\left(y^{(h)}, y^{(h-1)}\right)\right) .
\end{aligned}
$$

Thus, statistics might purely depend on the network $y^{(h)}$ (expressing structural patterns at time $t_{h}$ without a temporal component) or on both the network $y^{(h)}$ and $y^{(h-1)}$ (expressing dependence on the preceding observation). Note that statistics depending only on the previous network $y^{(h-1)}$ would lead to a non-identifiable model since their value is the same for all networks at time $t_{h}$.

\subsection{Stochastic actor-oriented models (SAOMs)}

Stochastic actor-oriented models (Snijders, 2001, 2005) assume that the observed networks $y^{(1)}, \ldots, y^{(T)}$ are snapshots of an underlying dynamic process driven by myopic actor decisions. Based on Markov assumption (2), this process is modeled separately for each interval $\left[t_{h-1}, t_{h}\right], 2 \leq h<T$, as a stochastic chain of tie flips (creating a new tie or dissolving an existing one) leading from $y^{(h-1)}$ to $y^{(h)}$. Changes do not occur synchronously, but successively on single ties. At each moment $t_{h-1}+\Delta t, 0 \leq$ $\Delta t<t_{h}-t_{h-1}$, change probabilities depend only on the current network structure $y$.

The models are actor-oriented in the sense that the tie flips are assumed to be performed by the actors, with each actor $i$, $1 \leq i \leq n$, controlling only his outgoing relations $y_{i j}$ to the $n-1$ other actors. When actor $i$ gets the opportunity to flip a tie in the current network $y$, he/she randomly chooses to change tie $y_{i j}$ into its opposite $1-y_{i j}$ with probability being proportional to the enhancement of his/her position in the resulting network. The enhancement of $i$ 's position when changing the tie $y_{i j}$ is measured by the evaluation function

$f_{i}\left(\theta^{(h)}, y, y^{-i j}\right)=\sum_{\ell=1}^{k} \theta_{\ell}^{(h)}\left(s_{i \ell}\left(y^{-i j}\right)-s_{i \ell}(y)\right)$,

where statistics $s_{i \ell}$ count certain local network configurations, like the number of outgoing ties, the number of reciprocated ties, or the number of transitive ties that actor $i$ holds. Assuming that the changing actors are uniformly chosen, the probability that $y_{i j}$ will be flipped in the next step is given by $\frac{1}{n} \frac{\exp \left(f_{i}\left(\theta^{(h)} y, y^{-i j}\right)\right)}{\sum_{k=1}^{n} \exp \left(f_{i}\left(\theta^{(h)} y, y^{-i k}\right)\right)}$. Note that not changing any tie, represented by term $\exp \left(f_{i}\left(\theta^{(h)}, y, y^{-i i}\right)\right)$, is also an option for actor $i$. 


\subsection{Experimental setup}

Temporal exponential random graph models and stochastic actor oriented models, in general, do not fall into the class of conditional independence models. For instance, including a statistic counting triangles in the dependent network implies that incident dyads are mutually dependent.

For each ERGM or SAOM statistic that introduces dependency among different ties, we define a lagged statistic that mimics the same structural effect while staying in the class of conditional independence models. Then we compare the quality of models that use only the lagged statistics (and therefore assume conditional independence) with their more general counterparts that use only the original ERGM respectively SAOM statistics.

Formally, for an ERGM statistic $s$ we define the associated lagged network statistic $x(y) \in \mathbb{R}^{n \times n}$ by setting for each pair $(i, j)$

$x_{i j}(y)=s\left(y^{+i j}\right)-s\left(y^{-i j}\right)$.

From this lagged network statistic we obtain a lagged ERGM statistic by setting

$s^{\prime}\left(y^{(h)}, y^{(h-1)}\right)=\sum_{i \neq j} y_{i j}^{(h)} \cdot x_{i j}\left(y^{(h-1)}\right)$,

i. e., by summing the values of $x$ computed at the preceding time $t_{h-1}$ over all pairs that are connected by a tie at time $t_{h}$. We then compare the quality of models that use the ERGM statistic $s$ with those that use the implied lagged statistic $s^{\prime}$. For instance, if the ERGM statistic $s$ counts transitive triangles then $s$ and $s^{\prime}$ assess the evolution in the top row of Fig. 1 in an identical way: the newly created tie from $A$ to $B$ closes a transitive triangle in $y^{(h)}$ (which is counted by $s$ ) but also closes a directed two path that was present in $y^{(h-1)}$ (which is counted by $s^{\prime}$ ). On the other hand, the evolution in the bottom row is differently assessed by $s$ and $s^{\prime}$ : the ERGM statistic $s$ counts one transitive triangle while the lagged statistic $s^{\prime}$ assumes a value of zero. A temporal ERGM that includes only statistics of the form given in Eq. (6) is a conditional independence model; in this case the model boils down to a binomial logit model for the occurrence of ties. Indeed, the conditional probability of a tie at time $t_{h}$ is then independent of the presence or absence of other ties at $t_{h}$ (albeit it might be dependent on other ties at $t_{h-1}$ ).

Analogously, given a SAOM statistic $s_{i}(y) \in \mathbb{R}, 1 \leq i \leq n$, the associated lagged network statistic $x(y) \in \mathbb{R}^{n \times n}$ is defined by setting for each pair $(i, j)$

$x_{i j}(y)=s_{i}\left(y^{+i j}\right)-s_{i}\left(y^{-i j}\right)$.

This lagged network statistic implies a lagged SAOM statistic by setting

$s_{i}^{\prime}\left(y, y^{(h-1)}\right)=\sum_{j=1}^{n} y_{i j} \cdot x_{i j}\left(y^{(h-1)}\right)$,

i.e., by summing the values of $x\left(y^{(h-1)}\right)$ over all outgoing ties of actor $i$ in the current network $y$. We then compare the quality of models that use the SAOM statistic $s$ with those that use the implied lagged statistic $s^{\prime}$.

\section{Application data and model specifications}

Comparison of the models is done with two datasets that differ, among others, in the length of the time interval between observation points. One of the two datasets is a friendship network among school children observed at four points in time with intervals of three months; the other dataset, the well-known Newcomb fraternity data (Newcomb, 1961), encodes a friendship network observed 15 times, once per week.
The first dataset, later referenced as Knecht (classroom) data, is the subset of the data collected by Knecht (2008) that is used in the Siena tutorial (Snijders, van de Bunt, \& Steglich, 2010b). ${ }^{1}$ The data is about a friendship network in a Dutch school class. Apart from the friendship data we use actor-level covariate data about the sex of the pupils and a dyadic covariate encoding whether two pupils were in the same primary school or not. One of the 26 pupils left this class between the second and the third observation point. Since the network quality measure that we consider in this paper depends on the size of the network we drop the leaving pupil from all four waves. Note that this level of missingness is very low compared to the levels considered in Huisman and Steglich (2008). ${ }^{2}$ Imputation of ties would be likely to favor those models that are most compatible with the rule of imputation (again see Huisman and Steglich (2008)).

The second dataset, the Newcomb (fraternity) data (Newcomb, 1961), encodes friendship among 17 men attending the University of Michigan in the fall of 1956 observed 15 times, once per week. The original data consists of preference rankings where each of the students ranks all 16 others from best friend to leastbest friend. We dichotomize this original data by keeping the top-four nominations for each actor without distinction of their ordering. Thus, the transformed data encodes the hypothetical answers to the question name your four best friends. Note that by this transformation the outdegrees are constant over the actors and over the different time steps. Furthermore, we apply other preprocessing rules that vary the time span between observations (to enable comparison with the Knecht classroom data) and that yield non-constant outdegrees (to assess whether this regularity affects our findings); see details in Section 5. The Newcomb data does not include any actor-level or dyad-level covariates.

Model specifications. Model estimation in the ERGM framework is done with the help of the $R$-package ergm (Handcock et al., 2011) which is part of the statnet package (Handcock, Hunter, Butts, Goodreau, \& Morris, 2003). For estimating the SAOMs, we use the R-package RSiena (Ripley \& Snijders, 2011). In both frameworks the specification is done by choosing the statistics $s_{\ell}$ that model the conditional probability of the network $y^{(h)}$, given the previous network $y^{(h-1)}$. We define and estimate three different models that we first describe intuitively. The basic model controls the edge density, inertia, and dependence on (actor- and dyad-)attributes. Two models extend the basic model and are compared with each other: The dyadicdependence model introduces reciprocity, indegree, outdegree, and transitivity effects. The conditional-independence model mimics the network effects of the dyadic-dependence model by substituting each statistic by its conditionally-independent counterpart, as it is described in Section 3.3. Our main question is whether the-statistically and computationally much simpler-conditional independence model performs as good as the dyadic-dependence model. Details are provided in the following.

(basic model) ERGMs control the density by the edges statistic that counts the number of edges in the network $y^{(h)}$; in SAOMs this is achieved by counting the number of outgoing ties of each actor. The lagged edges statistic is included in ERGMs and controls the inertia of ties from one time point to the next. Technically it is the ERGM statistic computed by Eq. (6) from the adjacency matrix

\footnotetext{
1 see http://www.stats.ox.ac.uk/ snijders/siena/tutorial2010_data.htm

2 To further assess the impact of dropping one actor we left out others, one by one, to obtain artificially reduced networks of 24 actors. The main result about the log-likelihood differences was never affected by this data reduction and parameter significance changed only in rare cases.
} 
of the previous wave. Thus, the lagged edges statistic counts the number of edges in $y^{(h)}$ that are also present in $y^{(h-1)}$. A lagged edges statistic is not necessary for SAOMs, since these model inertia implicitly by letting the sequence of tie changes start from network $y^{(h-1)}$. $^{3}$ All models contain four attribute-dependent statistics for the Knecht data (none for the Newcomb data): We use the main effects of the "sex" variable on outgoing and incoming ties (controlling whether boys tend to have more in-/out-edges than girls), the homophily effect of the "sex" variable (controlling whether pupils tend to have friends of the same sex), and a dyadic covariate term that counts those edges connecting pupils that were in the same primary school.

The dyadic-dependence model adds four statistics to the basic model. The mutual statistic counts the reciprocated ties in the network $y^{(h)}$. Furthermore, in the ERGM framework we include the statistics named gwesp ${ }^{4}$ and gwidegree for both datasets and additionally gwodegree for the Knecht data. ${ }^{5}$ We discuss the choice of the shape parameter (cf. Hunter \& Handcock, 2006) below. Within the SAOM framework we include the statistics named transitive ties and indegree popularity for both datasets and additionally outdegree activity for the Knecht data.

The conditional independence model substitutes the four additional statistics of the dyadic-dependence model by their conditionally-independent counterparts computed by the procedure described in Section 3.3.

Some technical details All ERGMs are estimated with the ergm $R$-package (Handcock et al., 2011) with an MCMC sample size set to 20,000 . The conditional independence models could in principle be estimated by ordinary logistic regression; this is not necessary since the estimation method of ergm recognizes the independence and automatically switches to a faster estimation procedure. To find appropriate shape parameters (cf. Hunter \& Handcock, 2006) for the statistics gwesp, gwidegree, and gwodegree we applied the following steps. We first estimated all models without fixing the shape parameters (i.e., by estimating curved exponential random graph models). For many time steps this resulted in poor convergence; for those steps where we did achieve good convergence, the shape parameter for the gwesp statistic was typically small but positive (around 0.1 ) and the estimated shape parameters for the gwidegree and gwodegree statistics were typically in the range $[0.4,0.8]$. We chose to fix the shape parameter for gwesp at 0.1 and for the other two at 0.5 . With this choice all models converged for all time steps.

All actor-oriented models were estimated with the maximum likelihood estimation method proposed in (Snijders, Koskinen, \& Schweinberger, 2010a) and implemented in the $R$-package RSiena (Ripley \& Snijders, 2011). For the majority of the models and time steps, convergence was excellent in terms of the criterion (proposed in Ripley and Snijders (2011)) of t-ratios for convergence less than 0.1 in absolute value, and for almost all models, convergence was still satisfying (indicated by t-ratios less than 0.2 ).

\footnotetext{
3 Including a lagged edges statistic in SAOMs results often in a non-convergent model.

4 gwesp stands for geometrically weighted edgewise shared partners.

5 The dichotomized Newcomb data has constant outdegree implying that the lagged gwodegree statistic is a constant multiple of the edges statistic-leading to a non-identifiable model.
}

\section{Results and discussion}

As outlined above, we compare ERG- and SAO-models that assume conditional independence with those that do not. The assessment criteria are, firstly, how well these models explain the observed data and, secondly, whether substantive findings about network effects on the evolution of ties are affected by the (potentially invalid) assumption of conditional independence.

\subsection{Conditional likelihood}

Since model parameters are estimated via maximum likelihood estimation, a natural way to quantify whether model $M$ explains the evolution from network $y^{(h-1)}$ to $y^{(h)}$ better than model $M^{\prime}$ is to compare the conditional probabilities of $y^{(h)}$, given $y^{(h-1)}$, i. e., ${ }^{6}$

$P_{M ; \theta}\left(Y^{\left(t_{h}\right)}=y^{(h)} \mid y^{(h-1)}\right)$ and $P_{M^{\prime} ; \theta^{\prime}}\left(Y^{\left(t_{h}\right)}=y^{(h)} \mid y^{(h-1)}\right)$,

where $\theta$ and $\theta^{\prime}$ are the maximum likelihood estimates when fitting the models $M$, respectively $M^{\prime}$, to the data $\left(y^{(h)} \mid y^{(h-1)}\right)$. Since we use the same pair of networks for model estimation and assessment, the conditional likelihoods can only be used to compare models with the same number of parameters. ${ }^{7}$ Two models are compared by the difference of the respective loglikelihoods ${ }^{8}$

$\log \left(P_{M ; \theta}\left(Y^{\left(t_{h}\right)}=y^{(h)} \mid y^{(h-1)}\right)\right)-\log \left(P_{M^{\prime} ; \theta^{\prime}}\left(Y^{\left(t_{h}\right)}=y^{(h)} \mid y^{(h-1)}\right)\right)$,

being positive when $M$ is the better model and negative when $M^{\prime}$ is better.

Concretely, we compare for each dataset and time step the dyadic-dependence model with the conditional independence model as described in Section 4. The differences in the loglikelihood are summarized graphically in Fig. 2, using timedependent bar charts. An upwards pointing black bar indicates that the dyadic-dependence model is better than the associated conditional-independence model; conversely, downwards pointing red bars indicate that the conditional independence model performed better. The height of a bar represents the magnitude of the difference, where the scaling on the vertical axis is such that the maximum difference is normalized with respect to data and model framework. ${ }^{9}$ Time advances from left to right, and for each estimation step there are two bars: differences in the SAOM-framework (slightly darker shades) are depicted to the right of differences in the ERGM-framework. Raw numbers are provided in Table 1.

In summary, dyadic-dependence models perform better than their conditionally-independent counterparts. In the Knecht data, with an inter-observation time of three months, there are no exceptions to this rule. On the other hand, the superiority of dyadicdependence models diminishes with shorter inter-observation time. In the Newcomb data the dyadic-dependence ERGMs improve over their conditionally-independent counterparts in seven out of 14 time steps; in the SAOM framework the dyadicdependence models show an advantage in ten out of 14 time steps.

\footnotetext{
6 For readability we denote with "model M" a class of ERGMs respectively SAOMs specified by a fixed selection of statistics; such a class of models is specialized to a concrete model by fixing the parameters.

7 Note that a model with $n(n-1)$ parameters could just remember the value of every tie variable in $y^{(h)}$ and, thus, "predict" the observed outcome with probability one.

8 Likelihoods of SAOMs are not directly computable. However, the data augmentation approach proposed in Snijders et al. (2010a) allows for estimating differences between log-likelihoods of two models.

9 We further transformed the absolute differences $d$ by setting the height proportional to $\log (d+1)$ which better reveals the sign of small values.
} 




Fig. 2. Upward pointing black bars indicate improvement in the log-likelihood when using dyadic-dependence models instead of associated conditional-independence models; downward pointing red bars indicate that the conditional-independence model performs better in the respective time step.

Table 1

Log-likelihood differences for both datasets (top row for ERGMs, bottom row for SAOMs). Positive values indicate a better performance of the dyadic-dependence models-indicated by an upward pointing bar in Fig. 2 .

\begin{tabular}{crrrrrrrrrrrrr}
\hline Knecht & & & & & & & & & & & & & \\
6.37 & 14.95 & 6.41 & & & & & & & & & & & \\
0.88 & 2.50 & 0.60 & & & & & & & & & & & \\
Newcomb & & & & & & & & & & & \\
-0.83 & -1.76 & 0.25 & 2.69 & -3.00 & 3.43 & -0.75 & -1.45 & 1.04 & -1.13 & 0.10 & -0.57 & 1.05 & 0.98 \\
0.03 & 0.04 & 0.15 & 0.32 & -0.08 & 0.33 & -0.03 & 0.11 & 0.12 & 0.16 & 0.02 & -0.02 & -0.16 & 0.18 \\
\hline
\end{tabular}

By the Markov assumption, the probability of the joint Newcomb data is

$P\left(y^{(2)}, \ldots, y^{(15)} \mid y^{(1)}\right)=P\left(y^{(2)} \mid y^{(1)}\right) \cdot P\left(y^{(3)} \mid y^{(2)}\right) \cdots P\left(y^{(15)} \mid y^{(14)}\right)$, where model parameters might change from interval to interval. Dyadic-dependence models achieve in both frameworks a (slightly) better joint likelihood: the sum of the log-likelihood differences is 0.045 for ERGMs and 1.175 for SAOMs.

Newcomb data with a longer time lag. How would conditional independence models have performed on the Newcomb data, if it had been collected with the same inter-observation time as the Knecht data? We might approach this question by separately fitting models to the pairs of networks $\left(y^{(13)} \mid y^{(1)}\right),\left(y^{(14)} \mid y^{(2)}\right)$, and $\left(y^{(15)} \mid y^{(3)}\right)$, ignoring the eleven intermediate observations..$^{10}$ Models allowing for dyadic-dependence outperformed conditional-independence models on this long-lagged Newcomb data. The log-likelihood differences are 3.93, 15.97, and 5.79 for ERGMs and 0.76, 1.25, and 0.35 in the SAOM framework. ${ }^{11}$

We further varied the preprocessing steps for the Newcomb data by considering only ties that are present in two consecutive waves. Therefore we defined a network $y^{(1 \& 2)}$ by intersecting the first two networks $y^{(1)}$ and $y^{(2)}$ as well as a network $y^{(14 \& 15)}$ by intersecting the last two networks $y^{(14)}$ and $y^{(15)} .^{12}$ These networks no longer have constant outdegrees (even though the outdegrees are constrained to be less than or equal to four). The resulting panel data is more comparable to the Knecht data with respect to the inter-observation time and the amount of network change. ${ }^{13}$ When fitting models to the data $\left(y^{(14 \& 15)} \mid y^{(1 \& 2)}\right)$ we found, again, that dyadic-dependence models outperform conditional independence models with a log-likelihood difference of 9.02 for ERGMs and 1.05 for SAOMs. Any variant of the long-lagged Newcomb data that we considered can be better explained by dyadic-dependence models. Results in this section, thus, suggest that the conditional independence assumption becomes more unrealistic with increasing inter-observation time.

\footnotetext{
10 The time difference between Wave 1 and 13 is 13 weeks (since there is a time gap of two weeks between Wave 9 and 10, due to a holiday break at that time) and, thus, roughly corresponds to three months.

11 Note that these differences are higher than any difference that results from the one-week steps in the respective model frameworks.

12 Thus, there is a tie from $i$ to $j$ in $y^{(182)}$ if and only if $y_{i j}^{(1)}=1$ and $y_{i j}^{(2)}=1$.

13 The Jaccard index (measuring the ratio of stable ties) is 0.304 , while the mean Jaccard index for the Knecht data is 0.412 . For comparison, the mean Jaccard index for the short-lagged Newcomb data is much higher, 0.681 .
}

\subsection{Sensitivity of derived network effects}

Another aspect on which we compare conditional-independence models with more general models are differences and similarities in derived significant network effects. The question is whether tests of hypothetical network effects are affected by the potentially invalid assumption of conditional independence. To shed light on this question we compare estimated parameters and significance levels for the conditional independence models and associated dyadic-dependence models described in Section 4.

Knecht data. Estimated parameters are shown in Table 2. In summary, conditional independence models yield nearly the same results for the attribute-based effects and for the assessment of reciprocity but lead to totally different conclusions for the other structural network effects.

More detailed, all models agree that in all time steps there is evidence for sex homophily but no significant parameters associated with the sex alter statistic (measuring whether boys receive more ties than girls). The sex ego effect (measuring whether boys tend to initiate more ties) is confirmed in the majority of cases and, with one exception, tie probabilities among pupils that have visited the same primary school are significantly increased in the second and third time step but not in the first.

The support for the reciprocity hypothesis (mutual statistic) is nearly unchallenged. With one exception, parameters are significantly positive across model frameworks and irrespective of whether models assume conditional independence or not.

The picture completely changes for the other structural network effects. While the dyadic-dependence ERGMs and SAOMs largely agree with each other, the conditional-independence models yield different conclusions. Tests with these models are in some cases more conservative (less rejection of the null hypothesis) and in other cases more liberal.

More detailed, the dyadic-dependence models yield in the majority of cases strong evidence for transitive closure and dependence on outdegree (activity ego), while their conditionallyindependent counterparts mostly fail to establish these effects. ${ }^{14}$ The disagreement is reversed when considering the popularity

14 Note that negative parameters associated with the ERGM statistics gwodegree and gwidegree point to preferential attachment with respect to outgoing, respectively incoming ties; cf. Hunter (2007). 
Table 2

Knecht classroom data. Comparison of estimated parameters of dyadic-dependence (DD) with conditionalindependence $(\mathrm{CI})$ models. For each block the parameters and their standard errors are listed from the first time step down to the third.

\begin{tabular}{|c|c|c|c|c|}
\hline & DD-ERGM & CI-ERGM & DD-SAOM & CI-SAOM \\
\hline Sex ego & $\begin{array}{l}0.581(0.274)^{*} \\
0.421(0.523)^{* * *} \\
0.731(0.269)^{*}\end{array}$ & $\begin{array}{l}0.582(0.319) \\
0.854(0.286)^{* * *} \\
0.939(0.291)^{* *}\end{array}$ & $\begin{array}{l}0.454(0.256) \\
0.618(0.226)^{* *} \\
0.477(0.201)^{*}\end{array}$ & $\begin{array}{l}0.324(0.267) \\
0.725(0.267)^{* * *} \\
0.593(0.206)^{* * *}\end{array}$ \\
\hline Sex alter & $\begin{array}{r}-0.432(0.325) \\
0.081(0.606) \\
-0.101(0.295)\end{array}$ & $\begin{array}{r}-0.344(0.323) \\
0.148(0.285) \\
0.274(0.290)\end{array}$ & $\begin{array}{r}-0.398(0.267) \\
0.071(0.250) \\
0.194(0.206)\end{array}$ & $\begin{array}{r}-0.345(0.274) \\
0.238(0.231) \\
0.351(0.226)\end{array}$ \\
\hline Sex homophily & $\begin{array}{l}0.676(0.263)^{*} \\
1.235(0.566)^{*} \\
0.978(0.253)^{* * *}\end{array}$ & $\begin{array}{l}0.898(0.340)^{* * *} \\
1.258(0.296)^{* * *} \\
1.162(0.298)^{* * *}\end{array}$ & $\begin{array}{l}0.678(0.268)^{*} \\
1.082(0.233)^{* * * *} \\
0.689(0.204)^{* * * *}\end{array}$ & $\begin{array}{l}0.741(0.286)^{* * *} \\
1.000(0.234)^{* * *} \\
0.810(0.212)^{* * *}\end{array}$ \\
\hline Primary school & $\begin{array}{c}-0.218(0.291) \\
0.770(0.484) \\
0.864(0.281)^{* * *}\end{array}$ & $\begin{array}{l}0.138(0.360)^{* * *} \\
1.070(0.313)^{*} \\
0.800(0.319)^{*}\end{array}$ & $\begin{array}{l}0.147(0.281) \\
0.568(0.237)^{*} \\
0.492(0.207)^{*}\end{array}$ & $\begin{array}{l}0.138(0.276) \\
0.587(0.248)^{*} \\
0.591(0.225)^{* *}\end{array}$ \\
\hline Mutual & $\begin{array}{l}1.737(0.396)^{* * * *} \\
1.396(0.786)^{* * * *} \\
1.253(0.361)^{* * *}\end{array}$ & $\begin{array}{l}1.376(0.347)^{* * * *} \\
0.728(0.306)^{*} \\
0.820(0.278)^{* * *}\end{array}$ & $\begin{array}{l}1.479(0.298)^{* * * *} \\
0.882(0.281)^{* * *} \\
0.913(0.198)^{* * * *}\end{array}$ & $\begin{array}{l}1.151(0.274)^{* * * *} \\
0.493(0.232)^{*} \\
0.626(0.193)^{* *}\end{array}$ \\
\hline Activity ego & $\begin{array}{l}-1.898(0.201)^{* * *} \\
-4.117(0.243)^{* * *} \\
-0.663(0.306)^{*}\end{array}$ & $\begin{array}{l}0.445(0.565) \\
1.048(0.513)^{*} \\
0.689(0.435)\end{array}$ & $\begin{array}{l}0.054(0.023)^{*} \\
0.102(0.018)^{* * *} \\
0.015(0.016)\end{array}$ & $\begin{array}{l}-0.028(0.026) \\
-0.025(0.023) \\
-0.025(0.016)\end{array}$ \\
\hline Popularity alter & $\begin{array}{r}0.357(0.912) \\
-1.254(1.596) \\
-0.229(0.997)\end{array}$ & $\begin{array}{l}-1.765(0.803)^{*} \\
-3.014(0.965)^{* *} \\
-0.425(0.940)\end{array}$ & $\begin{array}{l}0.089(0.047) \\
0.043(0.044) \\
0.008(0.041)\end{array}$ & $\begin{array}{l}0.221(0.060)^{* * * *} \\
0.082(0.041)^{*} \\
0.065(0.036)\end{array}$ \\
\hline Transitivity & $\begin{array}{l}0.569(0.025)^{* * * * *} \\
0.679(0.055)^{* * * *} \\
0.620(0.028)^{* * * *}\end{array}$ & $\begin{array}{l}0.333(0.164)^{*} \\
0.193(0.161) \\
0.108(0.222)\end{array}$ & $\begin{array}{l}0.723(0.237)^{* *} \\
0.594(0.353) \\
0.630(0.268)^{*}\end{array}$ & $\begin{array}{l}0.254(0.187) \\
0.123(0.172) \\
0.273(0.198)\end{array}$ \\
\hline
\end{tabular}

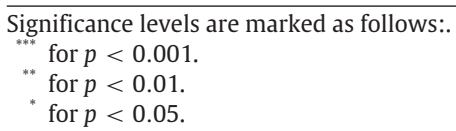

alter statistic (modeling the influence of indegree on the probabilities of ties). While the dyadic-dependence models consistently found this effect to be insignificant, conditional independence models reject the null hypothesis in the first two time steps.

The comparison so far suggests that conditional independence should not be assumed when testing structural network effects in the Knecht data; instead more general models based, for example, on the ERGM or SAOM frameworks, should be used.

Newcomb data. Results for the Newcomb fraternity data (Table 3) do not provide such clear distinction as for the Knecht classroom data. There is some disagreement between conditional independence models and their general counterparts, but-in accordance with our expectations-less serious than for the Knecht data. In contrast to what has been found on the Knecht data, there is also considerable disagreement between dyadic-dependence ERGMs and dyadic-dependence SAOMs.

More detailed, the transitivity statistic gwesp of the dyadicdependence ERGMs yields a significant parameter in nine of fourteen time steps-albeit with varying signs. In contrast, the three other models agree with each other by not rejecting the null hypothesis in any time step. There is more agreement with respect to the two other network effects. Parameters associated with the popularity alter statistic (dependence on indegree) have been found significant by all models in four time steps and, with some exceptions, mostly insignificant in the other intervals. Finally, the parameters of the mutual statistic (measuring reciprocity) are found to be significant by all models in three time steps and mostly insignificant in the others.

Altogether, the occasional disagreement between conditionalindependence models and their more general counterparts seems to be less severe in the Newcomb data than in the Knecht data. This provides further support to what we have found in the previous section: with increasing inter-observation times, the gap between dyadic-dependence and conditional independence models becomes more apparent.

\section{Conclusion}

The goal of this paper was to quantify the implications of assuming conditional independence of tie-change events when analyzing given longitudinal network data. The data characteristic that seems to be mostly responsible for the validity or invalidity of the conditional independence assumption is the inter-observation time. In the examples that we considered, three-months between observations yields data that seems to be inappropriate for conditional independence models. The more general dyadicdependence models consistently achieved a better likelihood for the Knecht data and the artificially thinned Newcomb data. This performance gap diminished in the shorter spaced Newcomb data. Note that both datasets encode network evolution among a group of pupils, respectively students, in the period after these meet for the first time-a situation that is likely to be characterized by relatively fast tie changes. In situations where ties are more stable, longer inter-observation times could still be consistent with the conditional independence assumption. In contrast, relations that change faster could yield network data with considerable intraobservation dependence, even if it is collected at a high frequency.

The question whether hypothetical network effects can alternatively be tested with conditional independence model gets a clear negative answer for the structural network effects (such as transitivity or preferential attachment) in the data with a three-month lag. Structural effects in the Knecht data are quite consistently assessed by the general ERGMs and SAOMs; in contrast, their conditionally independent counterparts lead to very different conclusions. This discrepancy gets somewhat attenuated in the Newcomb data with an inter-observation time of one week.

Tentatively we observe that there was considerable agreement across the two model frameworks, ERGMs and SAOMs, in the Knecht data but less in the Newcomb data. We are not aware of any studies that clarify whether temporal ERGMs and SAOM should confirm or reject the same network effects when applied 
Table 3

Newcomb data. Comparison of estimated parameters of conditional-independence (CI) models with dyadicdependence (DD) models. For each block the parameters and their standard errors are listed from the first time step down to the 14 th time step.

\begin{tabular}{|c|c|c|c|c|}
\hline & DD-ERGM & CI-ERGM & DD-SAOM & CI-SAOM \\
\hline \multirow{14}{*}{ Transitivity } & $0.278(0.046)^{* * *}$ & $0.160(0.177)$ & $0.252(0.287)$ & $0.084(0.216)$ \\
\hline & $0.138(0.048)^{* * *}$ & $0.157(0.190)$ & $0.358(0.315)$ & $0.214(0.244)$ \\
\hline & $-0.361(0.053)^{* * * *}$ & $0.216(0.230)$ & $-0.596(0.349)$ & $-0.083(0.297)$ \\
\hline & $0.780(0.061)^{* * * *}$ & $0.265(0.214)$ & $0.645(0.444)$ & $0.146(0.315)$ \\
\hline & $0.654(0.045)^{* * * *}$ & $0.382(0.222)$ & $0.208(0.335)$ & $0.317(0.273)$ \\
\hline & $0.019(0.052)$ & $-0.028(0.239)$ & $0.275(0.379)$ & $0.053(0.309)$ \\
\hline & $-0.042(0.058)$ & $-0.153(0.270)$ & $-0.681(0.433)$ & $-0.605(0.407)$ \\
\hline & $0.085(0.059)$ & $0.013(0.249)$ & $-0.180(0.437)$ & $0.027(0.342)$ \\
\hline & $0.053(0.059)$ & $0.087(0.271)$ & $0.410(0.452)$ & $0.533(0.389)$ \\
\hline & $-0.283(0.063)^{* * * *}$ & $0.166(0.254)$ & $-0.728(0.411)$ & $-0.135(0.326)$ \\
\hline & $0.004(0.047)$ & $0.296(0.212)$ & $0.141(0.345)$ & $0.106(0.305)$ \\
\hline & $-0.180(0.072)^{*}$ & $0.170(0.286)$ & $0.041(0.405)$ & $0.133(0.379)$ \\
\hline & $0.175(0.040)^{* * * *}$ & $-0.020(0.214)$ & $0.382(0.348)$ & $0.085(0.268)$ \\
\hline & $-0.176(0.050)^{* * * *}$ & $0.036(0.209)$ & $0.227(0.355)$ & $0.250(0.281)$ \\
\hline \multirow{14}{*}{ Popularity alter } & $-0.810(1.088)$ & $0.124(0.800)$ & $0.077(0.073)$ & $0.018(0.067)$ \\
\hline & $-1.284(1.103)$ & $-0.429(0.932)$ & $0.107(0.075)$ & $0.099(0.073)$ \\
\hline & $-1.257(1.235)$ & $-0.353(1.064)$ & $0.301(0.107)^{* * *}$ & $0.293(0.107)^{* * *}$ \\
\hline & $-1.626(0.818)^{*}$ & $-1.443(1.099)$ & $0.124(0.083)$ & $0.138(0.089)$ \\
\hline & $-1.179(0.781)$ & $-1.636(0.960)$ & $0.170(0.066)^{* * *}$ & $0.168(0.063)^{* * *}$ \\
\hline & $-3.719(0.899)^{* * * *}$ & $-2.630(0.959)^{* *}$ & $0.230(0.073)^{* * *}$ & $0.225(0.079)^{* * *}$ \\
\hline & $-0.457(0.990)$ & $-0.206(0.803)$ & $0.134(0.084)$ & $0.124(0.075)$ \\
\hline & $-2.232(0.912)^{*}$ & $-3.135(1.137)^{* * *}$ & $0.234(0.095)^{*}$ & $0.170(0.087)^{*}$ \\
\hline & $-1.663(0.923)$ & $-0.989(0.951)$ & $0.029(0.088)$ & $0.007(0.083)$ \\
\hline & $0.179(1.212)$ & $0.467(0.874)$ & $0.006(0.096)$ & $-0.025(0.084)$ \\
\hline & $-2.522(0.872)^{* * *}$ & $-2.845(1.387)^{*}$ & $0.238(0.076)^{* * *}$ & $0.289(0.088)^{* * *}$ \\
\hline & $-1.230(1.121)$ & $-0.117(1.028)$ & $0.002(0.087)$ & $0.011(0.082)$ \\
\hline & $-3.544(0.841)^{* * * *}$ & $-3.777(1.267)^{* * *}$ & $0.166(0.068)^{*}$ & $0.223(0.068)^{* * *}$ \\
\hline & $-1.744(0.869)^{*}$ & $-0.966(0.750)$ & $0.131(0.073)$ & $0.113(0.069)$ \\
\hline \multirow{14}{*}{ Mutual } & $0.369(0.440)$ & $0.824(0.388)^{*}$ & $0.685(0.344)^{*}$ & $0.762(0.334)^{*}$ \\
\hline & $1.962(0.497)^{* * * *}$ & $1.368(0.396)^{* * * *}$ & $1.248(0.358)^{* * * *}$ & $1.249(0.350)^{* * * *}$ \\
\hline & $0.889(0.514)$ & $0.589(0.469)$ & $1.072(0.471)^{*}$ & $0.659(0.463)$ \\
\hline & $0.758(0.544)$ & $0.836(0.470)$ & $1.147(0.490)^{*}$ & $0.995(0.478)^{*}$ \\
\hline & $0.803(0.502)$ & $0.602(0.447)$ & $0.730(0.388)$ & $0.713(0.379)$ \\
\hline & $0.923(0.550)$ & $0.544(0.504)$ & $0.805(0.459)$ & $0.373(0.448)$ \\
\hline & $1.112(0.558)^{*}$ & $0.835(0.546)$ & $0.554(0.613)$ & $0.693(0.611)$ \\
\hline & $-0.164(0.652)$ & $0.737(0.596)$ & $0.515(0.555)$ & $0.726(0.536)$ \\
\hline & $1.398(0.567)^{*}$ & $1.255(0.547)^{*}$ & $1.662(0.575)^{* *}$ & $1.348(0.562)^{*}$ \\
\hline & $-0.007(0.601)$ & $0.155(0.534)$ & $0.053(0.612)$ & $0.085(0.556)$ \\
\hline & $1.205(0.533)^{*}$ & $0.176(0.494)$ & $0.960(0.494)$ & $0.520(0.472)$ \\
\hline & $1.035(0.668)$ & $0.628(0.581)$ & $0.868(0.598)$ & $0.889(0.596)$ \\
\hline & $1.880(0.561)^{* * * *}$ & $1.447(0.459)^{* * *}$ & $1.324(0.432)^{* * *}$ & $1.483(0.427)^{* * * *}$ \\
\hline & $0.896(0.522)$ & $0.393(0.488)$ & $1.015(0.452)^{*}$ & $0.416(0.456)$ \\
\hline
\end{tabular}

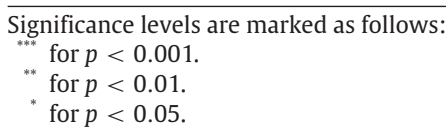

to the same data. A deeper comparison of ERGMs and SAOMs is certainly a promising task for future work. By design the two model frameworks make different assumptions. ERGMs assume that the network is in an equilibrium distribution. Although the structure of this equilibrium might be shaped by the previous network via lagged statistics, it is totally ignored how many tie-change events are needed to go from one observed network to the next. SAOMs, on the other hand, assume that there is an unobserved chain of events in which exactly one tie changes. In this framework there is no room for higher-order changes of mutually dependent tiechange events, e.g., events where three people meet each other in one single point in time or one actor synchronously cuts friendship to a group of actors.

Design for interval-censored event data. We mentioned in the introduction that the conditional independence assumption is not only an issue when dealing with panel data about relational states but also when analyzing interval-censored relational event data. We close our discussion by indicating that conditional independence in the latter data type can be analyzed with quite similar methods. As for panel data we can think of two model frameworks, one based on SAOMs and the other on ERGMs.
In the SAOM-like approach we can compare conditionalindependence models (like the one proposed by Brandes et al. (2009)) with more general models that assume an unobserved but existent order of all events in the same observation interval. It is then allowed that later events depend on previous ones, even if these happen in the same interval. Since the order of events is unobserved a more complicated model estimation has to be applied-similar to the maximum likelihood approach to SAOMs (Snijders et al., 2010a) that augments the data by simulated chains of change events.

In the ERGM-like approach all events in one observation interval are treated as one observed network. Thus, instead of considering the data from one interval as several single-tie events we treat it as one multi-tie event that is modeled by an ERGMlike distribution. This approach would require to dichotomize the so-constructed network event or to extend the ERGM framework to cases where ties are no longer binary variables but encode counting data. Designing such models and comparing them with event network models that assume conditional independence is another promising task for future work. 


\section{References}

Brandes, U., Lerner, J., \& Snijders, T.A.B. (2009). Networks evolving step by step: statistical analysis of dyadic event data. In Proc. 2009 Intl. Conf. Advances in Social Network Analysis and Mining, ASONAM 2009. (pp. 200-205).

Butts, C. T. (2008). A relational event framework for social action. Sociological Methodology, 38(1), 155-200.

Cranmer, S. J., \& Desmarais, B. A. (2011). Inferential network analysis with exponential random graph models. Political Analysis, 19(1), 66-86.

Crescenzi, M. J. C. (2007). Reputation and interstate conflict. American Journal of Political Science, 51(2), 382-396.

Frank, O., \& Strauss, D. (1986). Markov graphs. Journal of the American Statistical Association, 81(359), 832-842.

Handcock, M.S., Hunter, D.R., Butts, C.T., Goodreau, S.M., Krivitsky, P.N., \& Morris, M. (2011). Ergm: a package to fit, simulate and diagnose exponential-family models for networks. Version 2.4.3. URL http://statnetproject.org.

Handcock, M.S., Hunter, D.R., Butts, C.T., Goodreau, S.M., \& Morris, M. (2003). Statnet: software tools for the statistical modeling of network data. Version 2.0. URL http://statnetproject.org.

Hanneke, S., Fu, W., \& Xing, E. P. (2010). Discrete temporal models of social networks. Electronic Journal of Statistics, 4, 585-605.

Huisman, M., \& Steglich, C. (2008). Treatment of non-response in longitudinal network studies. Social Networks, 30, 297-308.

Hunter, D. R. (2007). Curved exponential family models for social networks. Social Networks, 29, 216-230.

Hunter, D. R., \& Handcock, M. S. (2006). Inference in curved exponential family models for networks. Journal of Computational and Graphical Statistics, 15(3), 565-583.
Knecht, A. (2008). Friendship selection and friends' influence. dynamics of networks and actor attributes in early adolescence. Ph.D. Thesis, University of Utrecht.

Maoz, Z., Terris, L. G., Kuperman, R. D., \& Talmud, I. (2007). What is the enemy of my enemy? Causes and consequences of imbalanced international relations, 1816-2001. Journal of Politics, 69(1), 100-115.

Newcomb, T. (1961). The acquaintance process. Holt, Reinhard \& Winston.

Ripley, R.M., \& Snijders, T.A.B. (2011). Manual for SIENA. Oxford: University of Oxford, Department of Statistics; Nuffield College. URL http://www.stats.ox.ac. uk/siena/.

Robins, G., Snijders, T., Wang, P., Handcock, M., \& Pattison, P. (2007). Recent developments in exponential random graph $\left(p^{*}\right)$ models for social networks. Social Networks, 29, 192-215.

Snijders, T. A. B. (2002). Markov chain Monte Carlo estimation in exponential random graph models. Journal of Social Structure, 3(2).

Snijders, T. A. B. (2001). The statistical evaluation of social network dynamics. In M. E. Sobel, \& M. P. Becker (Eds.), Sociological methodology. Basil Blackwell.

Snijders, T. A. B. (2005). Models for longitudinal network data. In P. J. Carrington, J. Scott, \& S. Wasserman (Eds.), Models and methods in social network analysis. Cambridge University Press.

Snijders, T. A. B., Koskinen, J., \& Schweinberger, M. (2010a). Maximum likelihood estimation for social network dynamics. Annals of Applied Statistics, 4, $567-588$.

Snijders, T. A. B., van de Bunt, G. G., \& Steglich, C. E. G. (2010b). Introduction to actorbased models for network dynamics. Social Networks, 32, 44-60.

Steglich, C. E. G., Snijders, T. A. B., \& Pearson, M. (2010). Dynamic networks and behavior: separating selection from influence. Sociological Methodology, 40(1), 329-393. 